\title{
MicroRNAs in Osteoclastogenesis and Function: Potential Therapeutic Targets for Osteoporosis
}

\author{
Xiao $\mathrm{Ji}^{\dagger}{ }^{\dagger}$, Xiang Chen ${ }^{\dagger}$ and Xijie $\mathrm{Yu}^{*}$ \\ Laboratory of Endocrinology and Metabolism, Department of Endocrinology, West China Hospital, \\ Sichuan University, 610041 Chengdu, China; jixiao0321@hotmail.com (X.J.); onlycx@163.com (X.C.) \\ * Correspondence: xijieyu@scu.edu.cn; Tel.: +86-28-8542-2362 \\ + These authors contribute equally to this work. \\ Academic Editors: Nalini Santanam and William Chi-shing Cho \\ Received: 5 February 2016; Accepted: 3 March 2016; Published: 9 March 2016
}

\begin{abstract}
Abnormal osteoclast formation and resorption play a fundamental role in osteoporosis pathogenesis. Over the past two decades, much progress has been made to target osteoclasts. The existing therapeutic drugs include bisphosphonates, hormone replacement therapy, selective estrogen receptor modulators, calcitonin and receptor activator of nuclear factor NF-kB ligand (RANKL) inhibitor (denosumab), etc. Among them, bisphosphonates are most widely used due to their low price and high efficiency in reducing the risk of fracture. However, bisphosphonates still have their limitations, such as the gastrointestinal side-effects, osteonecrosis of the jaw, and atypical subtrochanteric fracture. Based on the current situation, research for new drugs to regulate bone resorption remains relevant. MicroRNAs (miRNAs) are a new group of small, noncoding RNAs of 19-25 nucleotides, which negatively regulate gene expression after transcription. Recent studies discovered miRNAs play a considerable function in bone remodeling by regulating osteoblast and osteoclast differentiation and function. An increasing number of miRNAs have been identified to participate in osteoclast formation, differentiation, apoptosis, and resorption. miRNAs show great promise to serve as biomarkers and potential therapeutic targets for osteoporosis. In this review, we will summarize our current understanding of how miRNAs regulate osteoclastogenesis and function. We will further discuss the approach to develop drugs for osteoporosis based on these miRNA networks.
\end{abstract}

Keywords: microRNAs; osteoclasts; osteoclastogenesis; bone resorption; osteoporosis

\section{Introduction}

Osteoporosis, the growing metabolic skeletal disorder worldwide, has been called a "silent killer" due to its high rate of incidence and disability. Currently, drug selection for osteoporosis is limited. Bisphosphonates, the first-line drugs for osteoporosis due to their low price and high efficiency in reducing the risk of fracture, have gastrointestinal side-effects and, more importantly, long-term application of bisphosphonates inhibit the osteoblast and osteoclast functions simultaneously [1]. Bone formation and bone resorption are elaborate and well-coupled processes. The inhibition of bone resorption will lead to inhibition of bone formation and ultimately affect the efficacy of anti-bone-resorption drugs. Previous studies suggested that, except for coupling factors, the maintenance of osteoclast numbers is very important for keeping normal osteoblast functions. For example, bisphosphonates promote osteoclast apoptosis and decrease osteoclast numbers, which lead to inhibition of osteoblast functions. On the contrary, the application of inhibitors of cathepsin $\mathrm{K}$ and chloride channel 7 , which are all involved in bone resorption, have no effects on osteoblast functions, since these inhibitors only reduce bone resorption capacity but not osteoclast numbers. Abnormal osteoclast formation and resorption play a fundamental role in osteoporosis pathogenesis. Therefore, 
understanding osteoclast proliferation, differentiation, apoptosis, bone resorption, and the coupling mechanism between osteoclasts and osteoblasts, have a key role in the development of new drugs for osteoporosis.

MicroRNAs (miRNAs) are a class of small, noncoding RNAs of 19-25 nucleotides, which exist widely in eukaryotes and are highly conserved during biological evolution. After binding to $3^{\prime}$-untranslated regions (3'-UTR) within a target mRNA, miRNAs play a negative role in gene expression by regulating transcript localization, polyadenylation, and translation [2-4]. In 1993, lin-4RNAs were first discovered in Caenorhabditis elegans by Lee etc. [5]. In 2004, Chen et al. identified three miRNAs, which were not only specifically expressed in hematopoietic cells but the expression was dynamically modulated during early hematopoiesis and lineage commitment as well [6]. Since then, an increasing number of miRNAs have been identified to participate in osteoclast formation, differentiation, apoptosis, and resorption.

We have searched literature from PubMed and referred three other reviews [7-9]. This review aims to summarize our current understanding of how miRNAs regulate osteoclastogenesis and briefly refer to their potential clinical implications, such as biomarkers and the development of new drugs for osteoporosis based on these miRNA networks.

\section{Bone Remodeling and Osteoclasts}

Bone remodeling is a dynamic process throughout the whole lifetime of an individual, by which the skeleton maintains its structural integrity and exerts its metabolic functions as a repository of calcium and phosphorus [10]. Bone remodeling is regulated by the subtle equilibrium between osteoblastic bone formation and osteoclastic bone resorption. Firstly, there is an "activation" phase and a "resorption" phase. Cytokines are released at the site of remodeling to recruit osteoclasts to the bone surface. These osteoclasts form a ruffled border allowing them to adhere to the bone surface tightly. Between the osteoclast and the underlying bone, there exists a tiny isolated microenvironment into which the osteoclast's proton pump releases ions that create an acidic environment, making the mineralized component of the bone matrix dissolve. The organic matrix is exposed and degraded by cathepsin K [11]. Subsequently, the "reversal" phase begins. Mononuclear cells prepare the bone surface for osteoblasts and provide signals to recruit them. Along with proliferating, early osteoblasts secrete an extracellular matrix, which contains type I collagen abundantly. This matrix matures and is mineralized, and osteoblasts continue to differentiate. Finally, the bone surface is repaired. Those mature osteoblasts either undergo apoptosis, or eventually differentiate into osteocytes or bone surface lining cells [12].

Originating from mononuclear hematopoietic myeloid lineage cells, osteoclast precursors (OCPs) are formed in the bone marrow and subsequently attracted to the bloodstream by chemokines. Attracted by a variety of factors released from bone remodeling units (BRUs), OCPs are attracted back into bones and then they differentiate into osteoclasts [13]. During normal physiological conditions, osteoclastogenesis is regulated by osteoblasts and stromal cells, both of which provide two essential factors, macrophage colony-stimulating factor (M-CSF) and receptor activator of nuclear factor NF-KB ligand (RANKL). M-CSF plays an essential role in the survival, proliferation and the expression of RANK in early OCPs (monocyte/macrophage lineage). Therefore, the primary role of M-CSF is to provide survival signals during osteoclastogenesis [14]. In contrast, RANKL provides osteoclast differentiation signals and activates multiple signal transduction pathways, which turn on transcription factors NF- $\mathrm{kB}$, c-Fos, transcription factor nuclear factor of activated T cells (NFATc1), and microphthalmia-induced transcription factor (MITF) [15].

\section{Signaling Pathways of Osteoclast Differentiation}

Osteoclast differentiation is essentially modulated by three signaling pathways, which are activated by macrophage colony-stimulating factor (M-CSF), receptor activator of nuclear factor 
NF- $\mathrm{B}$ ligand (RANKL), and immunoreceptor tyrosine-based activation motif (ITAM), respectively (Figure 1).

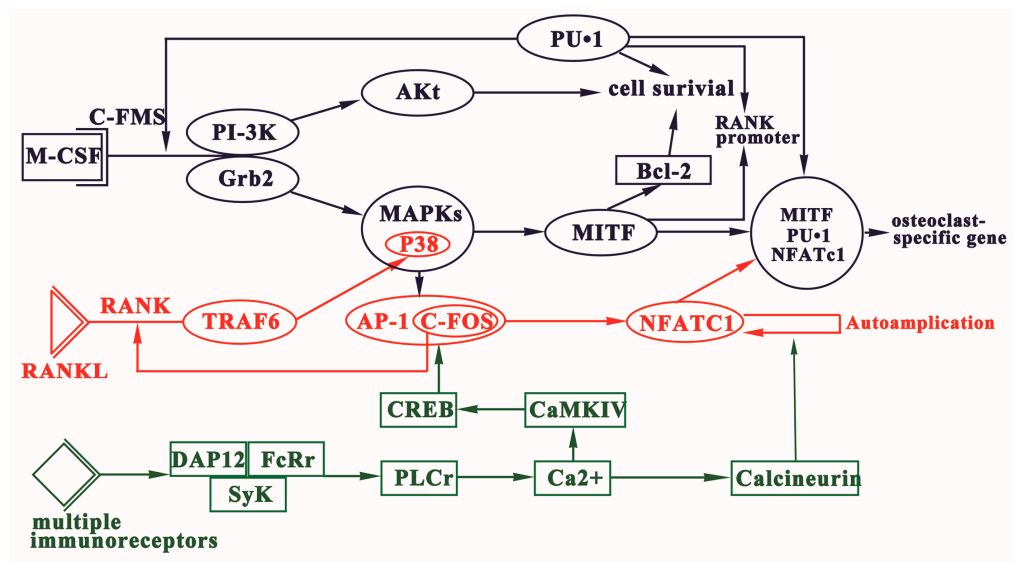

Figure 1. Three signaling pathways of osteoclast differentiation. Osteoclastogenesis is essentially modulated by three signaling pathways activated by M-CSF, RANKL, and ITAM. The binding of $\mathrm{M}-\mathrm{CSF}$ to its receptor c-Fms play function in the survival of osteoclast precursor cells. RANKL-RANK signaling commits the osteoclast precursors (OCPs) to osteoclast fate. ITAM-dependent co-stimulatory signals are imperative to RANKL-RANK signaling.

\subsection{Regulation of OCPs Formation via M-CSF Signaling}

M-CSF is pivotal for the survival and proliferation of OCPs. M-CSF, through its receptor c-Fms, transmits signals to the cell and then activates extracellular signal-regulated kinase (ERK) through Grb2 and phosphoinositide 3-kinase (PI-3K)/Akt $[16,17]$. The differentiation process of hematopoietic stem cells to OCPs is induced by transcription factors, such as purine-rich binding protein 1 (gene symbol: SPI1, PU.1) and Mitf [18]. PU.1 is a hematopoietic-specific member of the ETS family [19]. Deletion of PU.1 in mice results in a complete lack of OCPs leading to osteopetrosis [20]. There are PU.1 binding sites within promoters of many genes involved in osteoclast formation and function [21]. During the process of hematopoietic stem cells differentiating into the monocyte/macrophage lineage, PU.1 stimulates the expression of CSF1R which is the receptor of CSF1 (i.e., M-CSF) [22]. By upregulating the transcription factor c-FOS, CSF1R induces expression of receptor activator of NFKB (RANK; TNFRSF11A). In cooperation with other transcription factors, PU.1 modulates the RANK gene transcription [23]. Activator protein 1 (AP-1) plays a critical role in osteoclastogenesis. The AP-1 transcription factor complexes comprise of the Fra, Fos, Jun, and activating transcription factor (ATF) families.

MITF is another critical transcription factor participating in the late stages of osteoclastogenesis. Through a conserved mitogen-activated protein kinase (MAPK) consensus site, M-CSF induces phosphorylation of MITF [24]. Then MITF induces the expression of BCL-2 and promotes macrophage survival. Both $\mathrm{Mitf}^{\mathrm{mi} / \mathrm{mi}}$ and $\mathrm{Bcl} 2^{-/-}$mice suffer severe osteopetrosis [25]. Moreover, through binding to the sites within the RANK promoter, MITF and PU.1 increase RANK promoter activity three-fold and two-fold, respectively, and six-fold synergistically [26]. Conversely, Mitf-E levels are significantly upregulated by RANKL [27].

Both PU.1 and MITF not only play an important role in the survival of OCPs, but also participate in osteoclast-specific gene induction at the terminal stage of differentiation $[21,28]$.

\subsection{RANKL-RANK Signaling}

RANKL commits the OCPs to osteoclast fate. The activation of RANKL-RANK signaling leads to the expression of genes involving the fusion of mononuclear osteoclast precursors, like dendritic cell-specific transmembrane protein (DC-STAMP), as well as of genes regulating resorption capacity of 
multinucleated osteoclasts, including cathepsin K, chloride channel 7, matrix metalloprotein 9, and calcitonin receptor.

RANKL-RANK binding recruits TRAF-6 to activate PI-3K, NF- $\mathrm{KB}$ family of transcription factors and all three MAPK pathways, including ERK, JNK (Janus N-terminal kinase), and p38. NF-kB is required for the expression of a variety of cytokines, including IL-6, IL-1, TNF- $\alpha$, GM-CSF, RANKL, and other growth factors. Protein kinase p38 is activated via phosphorylation of MAPK kinase (MKK) 6. The activation of p38 results in the downstream activation of MITF [29]. Hence, MITF exists downstream of the M-CSF and RANKL signaling pathways. Treatment with the p38 inhibitors increases phosphorylation of ERK, showing a balance between ERK and p38 phosphorylation.

RANKL induces the expression of the AP-1 complexity, which consists of Fos (c-Fos, FosB, Fra-1, Fra-2) and Jun (c-Jun, JunB, JunD) [30]. NFATc1 expression is dependent on the TRAF-6-NF- $k B$ and c-Fos pathways, which are activated by both RANKL and $\mathrm{Ca}^{2+}$ signaling. NFATc1 regulates OC-specific genes, such as tartrate resistant acid phosphatase (TRAP) [31], Cathepsin K [21], calcitonin receptor, osteoclast-associated receptor (OSCAR) [32], and $\beta 3$ integrin [33].

\subsection{Immunoreceptor Tyrosine-Based Activation Motif (ITAM)-Dependent Costimulatory Signals}

M-CSF and RANKL are not sufficient to activate the signals required for osteoclastogenesis. Immunoreceptor tyrosine-based activation motif (ITAM)-dependent costimulatory signals, activated by multiple immunoreceptors, are essential for osteoclastogenesis. Both Fc receptor common $\gamma$ subunit $(\mathrm{F} C \mathrm{R} \gamma)$ and DNAX-activating protein 12 (DAP12) are ITAM-harboring adapters. In osteoclast precursor cells, FcR and DAP12 associated with multiple immunoreceptors activate calcium signaling through phospholipase $C$ [34]. These receptors include OSCAR, triggering receptor expressed in myeloid cells-2 (TREM-2), signal-regulatory protein $\beta 1$ (SIRP $\beta 1$ ), and paired Ig-like receptor-A (PIR-A). These receptor-mediated signals cannot substitute RANKL but act with RANKL cooperatively. Therefore, ITAM-mediated signals can be identified as co-stimulatory signals for RANK.

\section{4. miRNAs in Osteoclasts}

Osteoclast differentiation is regulated by transcriptional, post-transcriptional, and post-translational mechanisms. miRNAs are fundamental post-transcriptional regulators of gene expression. miRNAs play a key role in the normal bone development. Heterozygous microdeletions in the MIR17HG locus, encoding microRNA 17-92 cluster, lead to autosomal dominant Feingold syndrome in humans, characterized by short stature, microcephaly, and abnormal development of fingers and toes [35]. Further study on animal models carrying targeted deletions of individual components of miR-17 92 revealed that miR-17 seed family is critical in patterning of the axial skeleton [36]. MicroRNA-related single nucleotide polymorphisms (SNPs) also have a potential impact on the skeletal phenotype [37]. Furthermore, emerging evidence suggests that miRNAs are involved in the multiple biological and pathological processes in osteoclast proliferation, differentiation, apoptosis, cytoskeleton formation, and bone resorption. During the early, middle, and late stages of murine osteoclastogenesis, miRNA microarray analysis showed 49 miRNAs were upregulated and 44 were downregulated [38]. In the following section, we will discuss relevant miRNAs in osteoclasts and their potential targets and signaling pathways (Table 1 and Figure 2).

Table 1. Summary of miRNAs, their target genes, expression profile, and effects on osteoclasts.

\begin{tabular}{|c|c|c|c|c|c|}
\hline miRNAs & Sample Resources & Target Gene (s) & Expression & Function & References \\
\hline $\operatorname{miR}-29$ & BMMs, RAW264.7 & $\begin{array}{c}\text { CDC42, SRGAP2, } \\
\text { NFIA, CD93, } \\
\text { CALCR }\end{array}$ & $\uparrow$ & promotes & [43] \\
\hline miR-31-5p & BMMs & RhoA & $\bar{\uparrow}$ & promotes & [44] \\
\hline
\end{tabular}


Table 1. Cont.

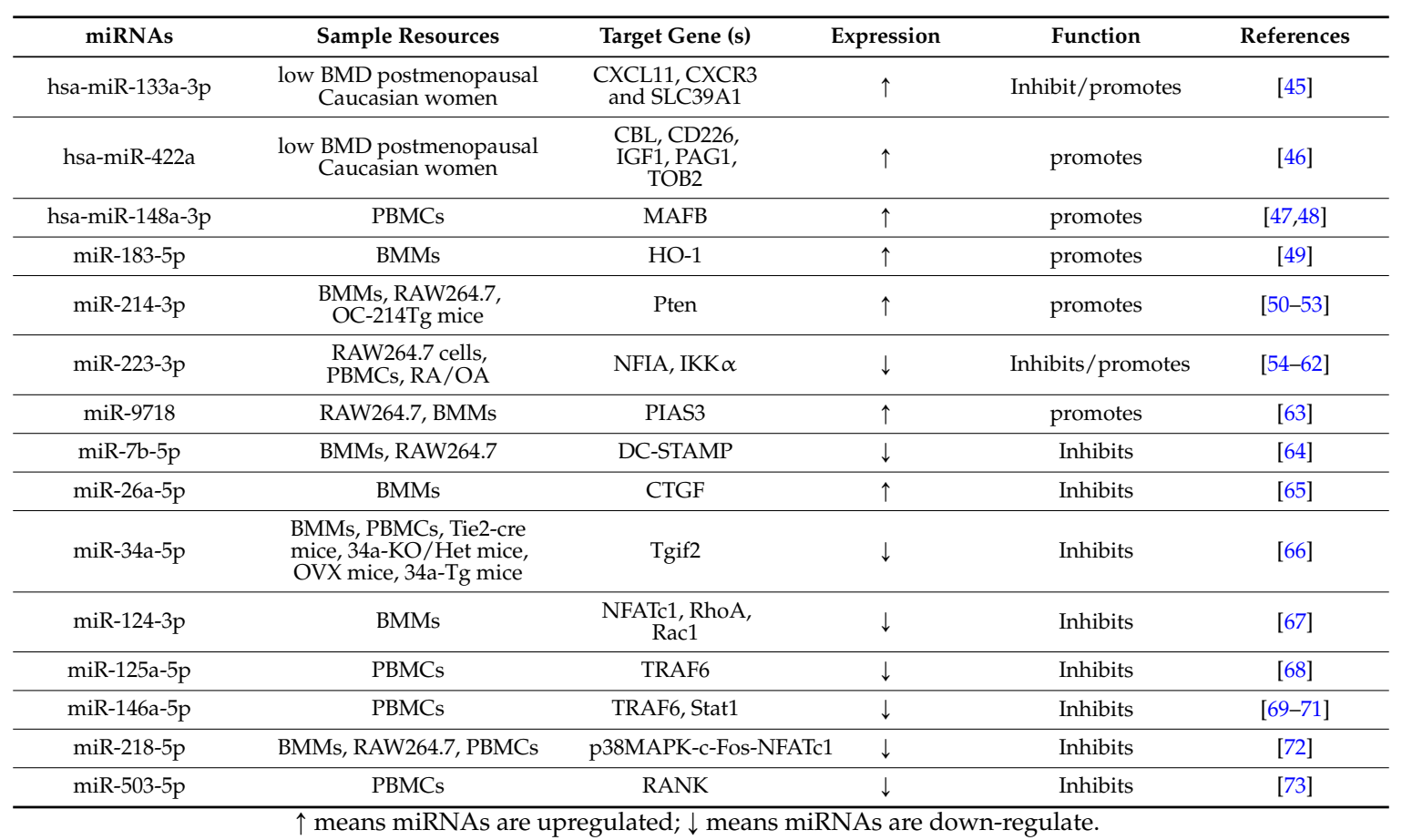

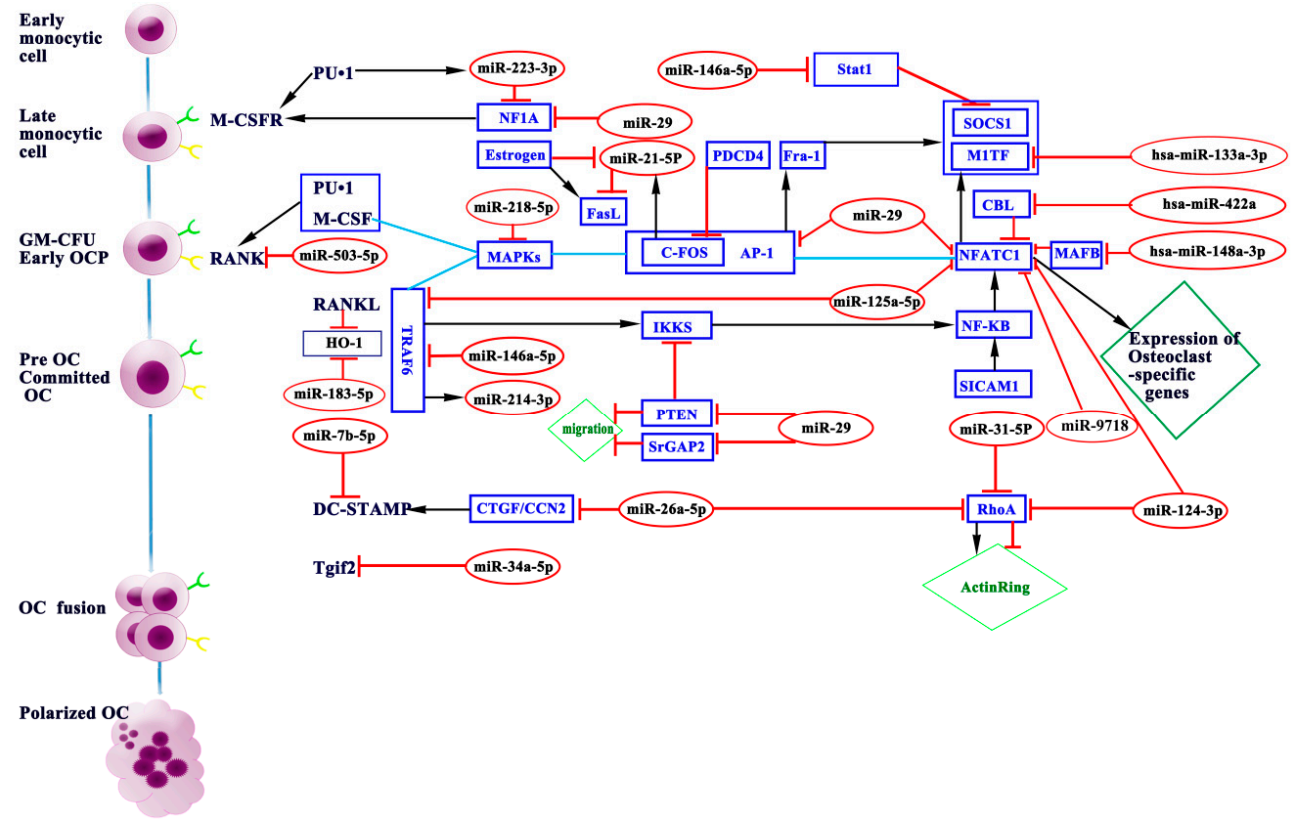

Figure 2. miRNAs modulate osteoclast differentiation and function. Through repressing varieties of target genes, miRNAs participate in osteoclast differentiation and maturation. Blue boxes indicate targets of miRNAs. Red ovals indicate miRNAs involved in osteoclastogenesis. Green receptors indicate M-CSFR, and yellow receptors indicate RANK.

\section{1. miRNAs Promoting Osteoclastogenesis}

\subsection{1. $\mathrm{miR}-21-5 \mathrm{p}$}

miR-21-5p is highly expressed in osteoclast precursors, and its expression levels are upregulated during RANKL-induced osteoclastogenesis [39]. miR-21-5p simultaneously promotes 
osteoclastogenesis and induces osteoclastic apoptosis. Transcription factors for osteoclastogenesis, such as c-Fos and PU.1, trigger miR-21-5p transcription via binding to the promoter of miR-21-5p [40]. miR-21-5p downregulates programmed cell death 4 (PDCD4) protein levels and, thus, removes its inhibitory effect on c-Fos. At the same time, RANKL-induced c-Fos upregulates miR-21-5p expression. Therefore, c-Fos/miR-21-5p/PDCD4 forms a positive feedback loop, promoting RANKL-induced osteoclastogenesis [39]. In addition, miR-21-5p plays a critical role in estrogen-related osteoclastogenesis. Estrogen inhibits osteoclastogenesis and induces osteoclastic apoptosis [41]. Estrogen attenuates miR-21-5p biogenesis; therefore, the protein levels of FasL (Fas ligand), a target of miR-21-5p, are post-transcriptionally increased, which induces osteoclastic apoptosis [42].

\subsection{2. $\mathrm{miR}-29$}

miR-29 family includes miR-29a-3p, miR-29b-3p, and miR-29c-3p. During osteoclastogenesis from bone marrow monocytes (BMMs) and monocytic cell line RAW264.7, all miR-29 family members increased, which was consistent with the expressions of osteoclast markers TRAP and cathepsin K [43]. Knockdown of miR-29 inhibited commitment and migration of pre-osteoclasts without interfering cell viability, actin ring formation, or apoptosis in mature osteoclasts [43]. The target genes of miR-29 family are Calcr (calcitonin receptor), RNAs critical for cytoskeletal organization, including Cdc42 (cell division control protein 42) and Srgap2 (SLIT-ROBO Rho GTPase-activating protein 2), and those associated with the macrophage lineage, including Gpr85 (G protein-coupled receptor 85), Nfia (nuclearfactor I/A), and Cd93 [43].

\subsection{3. $\mathrm{miR}-31-5 \mathrm{p}$}

miR-31-5p was highly upregulated during osteoclastogenesis under RANKL induction [44]. Inhibition of miR-31-5p attenuated the RANKL-induced osteoclast formation and bone resorption [44]. Further study suggested that actin ring formation in osteoclasts was severely impaired by miR-31-5p inhibition. RhoA, a target gene of miR-31-5p, was upregulated by miR-31-5p inhibition [44]. RhoA plays a key role in actin ring formation in osteoclasts. Therefore, miR-31-5p is a positive regulator of osteoclast formation and bone resorption through targeting RhoA.

\subsubsection{Hsa-miR-133a-3p and Hsa-miR-422a}

The expression of hsa-miR-133a-3p and hsa-miR-422a was upregulated in circulating monocytes of low bone mineral density (BMD) post-menopausal Caucasian women [46,47]. Bioinformatic analysis revealed that three osteoclast-related genes to be target for hsa-miR-133a-3p [45], including CXCL11, CXCR3, and SLC39A1; however, the negative correlations between hsa-miR-133a-3p and all three genes had no statistical significance using Pearson correlation analysis [45]. Bioinformatic analysis also predicted several potential target genes for hsa-miR-422a [46]. Negative correlations between hsa-miR-422a and five of these genes (CBL, CD226, IGF1, PAG1, and TOB2) had statistical significance [46]. Therefore, both hsa-miR-133a-3p and hsa-miR-422a may act as potential microRNA biomarkers for post-menopausal osteoporosis.

\subsubsection{Hsa-miR-148a-3p}

miRNA expression profile analysis showed miR-148a-3p was dramatically upregulated during osteoclastogenesis from human CD14+ peripheral blood mononuclear cells (PBMCs) using microarray [47]. Overexpression of hsa-miR-148a-3p in CD14+ PBMCs facilitated osteoclastogenesis, whereas inhibition of hsa-miR-148a-3p depressed it [47]. V-maf musculoaponeurotic fibrosarcoma oncogene homolog B (MAFB) is a target of hsa-miR-148a-3p, which negatively regulates RANKL-induced osteoclastogenesis [47]. Therefore, hsa-miR-148a-3p acts as a positive regulator of osteoclastogenesis through inhibiting MAFB. The higher expression level of hsa-miR-148a-3p in CD14+ PBMCs from lupus patients may play a role in the enhanced osteoclastogenesis and lower BMD $[47,48]$. 


\subsection{6. $\mathrm{miR}-183-5 \mathrm{p}$}

miR-183-5p is a member of miR-182-183 cluster located on the 7q31-34. This cluster includes miR-96, miR-182 and miR-183 [49]. miR-183-5p expression is upregulated during RANKL-induced osteoclastogenesis from BMMs. Inhibition of miR-183-5p led to a substantial reduction in TRAP positive multinucleated OCs [49]. Heme oxygenase-1 (HO-1) is predicted to be a target of miR-183-5p. RANKL decreased the expression of HO-1 and stimulated osteoclastogenesis. miR-183-5p inhibitor increased the expression of HO-1 and decreases osteoclast differentiation [49]. Therefore, miR-183-5p is a positive regulator of osteoclastogenesis through inhibiting HO-1 expression.

\subsection{7. $\mathrm{miR}-214-3 \mathrm{p}$}

miR-214-3p is highly conserved across vertebrates and has been suggested to play an important role in vertebrate skeletal development [50]. miR-214-3p is upregulated during osteoclastogenesis from BMMs induced by M-CSF and RANKL [51]. Overexpression of miR-214-3p in BMMs facilitates osteoclastogenesis, whereas inhibition of miR-214-3p depresses it [51]. Phosphatase and tensin homolog (PTEN), a tumor suppressor, negatively regulates PI3K/Akt signaling as a lipid phosphatase [52]. PTEN plays an essential role in regulating cell proliferation, migration, invasion, and apoptosis [52]. Phosphorylated PTEN reduces osteoclastogenesis by inhibiting the PI3K/Akt pathway [53]. Zhao et al. suggested that miR-214-3p regulates osteoclastogenesis through targeting the PTEN/PI3k/Akt pathway [51].

\subsection{8. $\mathrm{miR}-223-3 \mathrm{p}$}

miR-223-3p has been well-studied in osteoclastogenesis. Like miR-21-5p, miR-223-3p is downregulated during osteoclast differentiation [54]. There are conflicting studies indicating that miR-223-3p is capable of enhancing or suppressing osteoclast differentiation [55]. Antisense to miRNA-223 significantly suppressed osteoclast differentiation and osteoclast bone resorbing activity [56]. However, overexpression of pre-miR-223 also completely blocked TRAP-positive osteoclast formation, indicating that appropriate miRNA-223 expression levels should be kept during osteoclastogenesis [57].

miR-223-3p is specifically expressed in CD11b positive myeloid cell lineages [6]. By inhibiting NFIA (transcriptional repressor Nuclear Factor IA) expression, miR-223-3p regulates osteoclast differentiation. NFIA, a CCAAT-box binding transcription factor, is a member of the dimeric DNA-binding nuclear factors I (NFI) protein family [58]. miR-223-3p binds to specific sites within the promoter of NFIA and represses transcription by influencing epigenetic events [59]. In turn, NFIA and $\mathrm{C} / \mathrm{EBP} \alpha$ compete for binding to the miR-223-3p promoter, maintaining miR-223-3p expression levels [60]. When miR-223-3p expression is extremely low, NFIA will be upregulated, thus blocking osteoclast differentiation.

PU.1, miR-223-3p, NFIA and macrophage colony-stimulating factor receptor (M-CSFR) form a positive feedback loop. PU.1 upregulates miRNA-223 expression and then miRNA-223 downregulates NFIA expression, resulting in upregulation of M-CSFR [56]. In conflict with the above PU.1/miR-223-3p/NFIA positive feedback theory, miR-223-3p overexpression was reported to block osteoclast differentiation in RAW264.7 cells [57] and peripheral blood mononuclear cells (PBMCs) [62]. IKK $\alpha$, a critical factor in the NF-KB pathway, is downregulated by miR-223-3p for inhibiting differentiation of osteoclasts [7]. Through excess p52 in the absence of RelB, the non-canonical NF-KB pathway involved in IKK $\alpha$, could be one explanation for the dual role of miR-223-3p in osteoclast differentiation [61].

\subsection{9. $\mathrm{miR}-9718$}

miR-9718 is highly conserved between human and mouse, which is preferentially expressed in bone tissue, especially in osteoclasts [63]. The expression level of miR-9718 significantly increased during osteoclastogenesis from BMMs and RAW264.7 cells. Upregulation of miR-9718 in RAW264.7 
cells facilitated M-CSF and RANKL-induced osteoclastogenesis, whereas inhibition of miR-9718 repressed it [63]. PIAS3 is a target of miR-9718 [63]. PIAS3 belongs to protein inhibitor of activated STAT (PIAS) family, which consists of PIAS1, PIAS3, PIASx, and PIASy. PIAS3 inhibits osteoclastogenesis through downregulating NFATc1 and OSCAR [63]. Therefore, miR-9718 acts as a positive regulator of osteoclastogenesis by targeting PIAS3.

\section{2. miRNAs Inhibiting Osteoclastogenesis}

\subsection{1. $\mathrm{miR}-7 \mathrm{~b}-5 \mathrm{p}$}

miR-7b-5p was markedly downregulated during osteoclastogenesis from RAW264.7 cells induced by M-CSF and RANKL. Overexpression of miR-7b-5p in RAW264.7 cells decreased the numbers of TRAP-positive multinucleated cells, whereas inhibition of miR-7b-5p enhanced osteoclastogenesis [64]. DC-STAMP is predicted to be a target of miR-7b-5p. DC-STAMP is a key regulator of cell-cell fusion for the formation of mature osteoclast. Therefore, miR-7b-5p is a negative regulator of osteoclastogenesis through inhibiting DC-STAMP and its downstream signal factors including NFATc1, c-Fos, Akt, Irf8, Mapk1, and Traf6 [64].

\subsection{2. $\mathrm{miR}-26 \mathrm{a}-5 \mathrm{p}$}

miR-26a-5p is an important regulator of cell proliferation and differentiation. The miR-26a-5p expression was upregulated at the late stage of osteoclastogenesis induced by RANKL [65]. Expression of an miR-26a-5p mimic in osteoclast precursor cells attenuated osteoclastogenesis by suppressing the expression of connective tissue growth factor/CCN family 2 (CTGF/CCN2), while inhibitor of miR-26a-5p enhanced RANKL-induced osteoclastogenesis as well as CTGF expression [65]. CTGF can promote osteoclastogenesis via upregulation of DC-STAMP. Therefore, miR-26a-5p is a negative regulator of osteoclastogenesis by targeting CTGF [65].

\subsection{3. $\mathrm{miR}-34 \mathrm{a}-5 \mathrm{p}$}

miR-34a-5p is highly conserved between mouse and human. The expression of miR-34a-5p is downregulated during osteoclast differentiation [66]. One miR-34a-5p precursor (pre-miR-34a) significantly depressed osteoclast differentiation both from mouse BMMs and from PBMCs [66]. Osteoclastic miR-34a-5p-overexpressing transgenic mice showed lower bone resorption and higher bone mass. On the contrary, miR-34a-5p knockout and heterozygous mice exhibited augmented bone resorption and lower bone mass [66]. Mechanistically, transforming growth factor-b-induced factor 2 (Tgif2), a novel regulator of osteoclastogenesis by stimulating the activity of NFATc1, NF- $\mathrm{kB}$, and c-Jun, has been demonstrated to be a target of miR-34a-5p [66]. Therefore, miR-34a-5p serves as a negative regulator of osteoclastogenesis by inhibiting Tgif2.

\subsection{4. $\mathrm{miR}-124-3 \mathrm{p}$}

miR-124-3p has been suggested to have a putative tumor-suppressive role. Previous study indicated it also may be an intrinsic negative regulator of osteoclast differentiation by suppressing NFATc1 expression. NFATc1 is a key regulator of osteoclastogenesis. TargetScan, a web-based bioinformatics tool, predicted two conserved binding sequences of miR-124-3p in the $3^{\prime}$ UTR region of mouse NFATc1 gene. During the osteoclastic differentiation from BMMs induced by RANKL, the expression of miR-124-3p rapidly decreased. Pre-miR-124 significantly inhibited the RANKL-induced NFATc1 induction and osteoclast differentiation. On the contrary, inhibition of miR-124-3p potently promoted NFATc1 expression and osteoclastogenesis. miR-124-3p also reduces the expression of RhoA and Rac1, through which it might inhibit the migration of osteoclast precursors [67]. 


\subsection{5. $\mathrm{miR}-125 \mathrm{a}-5 \mathrm{p}$}

miR-125a-5p played a negative function in osteoclastogenesis through a novel TRAF6/NFATc1/miR-125a-5p regulatory feedback loop [68]. During M-CSF and RANKL-induced osteoclastogenesis from circulating CD14+ PBMCs, miR-125a-5p was dramatically downregulated [68]. Overexpression of miR-125a-5p in CD14+ PBMCs inhibited osteoclastogenesis, and vice versa [68]. Overexpression of NFATc1 inhibited miR-125a-5p transcription [68].

\subsection{6. miR-146a-5p}

miR-146a-5p, a negative regulator of immune and inflammatory responses, is upregulated in rheumatoid arthritis (RA) synovium and PBMCs [70]. miR-146a-5p directly targets signal transducer and activator transcription 1 (Stat1), and Stat1 selectively attenuates of SOCS1 [71]. Yao et al. successfully transported miR-146a into human PBMCs and subsequently demonstrated the inhibitory function of miR-146a in osteoclastogenesis [69].

\subsection{7. $\mathrm{miR}-218-5 \mathrm{p}$}

miR-218-5p has been demonstrated to stimulate bone formation. miR-218-5p has also been suggested to be a negative regulator of osteoclastogenesis. The expression of miR-218-5p was decreased in CD14+ PBMCs from post-menopausal osteoporosis patients compared with healthy control. During osteoclastogenesis from BMMs and RAW264.7 induced by RANKL, miR-218-5p expression was significantly downregulated. Upregulation of miR-218-5p obviously inhibited the formation of multinuclear osteoclasts, the migration of osteoclast precursors, actin ring formation, and bone resorption along with the decreased TRAP and Cathepsin K expression. Mechanistically, miR-218-5p suppresses osteoclastogenesis by targeting the p38MAPK-c-Fos-NFATc1 pathway [72].

\subsection{8. $\mathrm{miR}-503-5 \mathrm{p}$}

miR-503-5p has also been found to be downregulated in CD14+ PBMCs from post-menopausal osteoporosis patients compared with healthy controls [73]. miR-503-5p, located on Xq26.3, facilitates monocytic differentiation by targeting cell-cycle regulators. Overexpression of miR-503-5p in CD14+ PBMCs inhibited RANKL-induced osteoclastogenesis. Conversely, silencing miR-503-5p in CD14+ PBMCs promoted osteoclastogenesis [73]. Mechanistically, miR-503-5p acts as a negative regulator of osteoclastogenesis through inhibiting RANK, which was confirmed to be a target of miR-503-5p [73].

\section{Potential Clinical Implications of miRNAs and miRNAs-Based Therapeutic Strategy for Osteoporosis}

The existing therapeutic drugs include bisphosphonates, hormone replacement therapy, selective estrogen receptor modulators, calcitonin, and RANK ligand inhibitor (denosumab), etc. Bisphosphonates are nowadays the first-line anti-resorptive medication. There exist several potential adverse clinical events concomitant with the medication of bisphosphonates, including osteonecrosis of the jaw [74], atrial fibrillation [75], acute inflammatory response [75], and oversuppression of bone turnover [76]. Bone formation and bone resorption are well-coupled processes. The inhibition of bone resorption will result in inhibition of bone formation [1].

miRNAs are involved in the osteoclast proliferation, differentiation, cell-fusion, apoptosis, cytoskeleton formation, and bone resorption, which were summarized in Table 1 and Figure 2. As stated above, most miRNAs are involved in promoting or inhibiting osteoclast formation and maturation. Only a few miRNAs are capable of affecting osteoclast function.

\subsection{Potential Use of miRNAs as Biomarkers}

The role of miRNAs as biomarkers for bone diseases has drawn much attention recently. 


\subsubsection{Biomarkers for Osteoclasts Activity}

As a positive regulator in osteoclast formation, the expression of miR-29 (a/b/c) family was upregulated during osteoclastogenesis in vitro cell culture, which was consistent with the expression of osteoclast markers TRAP and cathepsin K [43]. In contrast, upregulation of miR-218-5p, a negative ocstoclastogenesis regulator, was consistent with the decreased TRAP and Cathepsin K expression [72]. The expression of plasma TRAP5 $b$ has been used to estimate the activity of osteoclasts; thus, miR-29 and miR-218-5p need further study to validate their potential as novel biomarkers for osteoclasts activity.

Panache et al. discovered miR-21-5p expression was correlated with bone resorption marker CTX [77].

\subsubsection{Biomarkers for Post-Menopausal Osteoporosis}

Both miR-133a-3p and miR-422a were upregulated in human circulating monocytes from the lower BMD post-menopausal Caucasian women groups [45,46]. In contrast, the expression of miR-218-5p and miR-503-5p was decreased in CD14+ PBMCs from post-menopausal osteoporosis patients compared with healthy controls [72,73]. Therefore, miR-133a-3p, miR-422a, miR-218-5p, and miR-503-5p have potential acting as biomarkers for post-menopausal osteoporosis.

Panache et al. discovered miR-122-5p, miR-125b-5p, and miR-21-5p were upregulated in serum from osteoporotic fracture patients compared with osteoarthritic controls [77]. Li et al. have validated downregulation of miR-21-5p and upregulation of miR-133a-3p in the plasma from osteoporosis and osteopenia patients versus normal Chinese post-menopausal women. Additionally, the circulating expression levels of miR-21-5p and miR-133a-3p were found to be correlated with the BMD [78].

Seeliger et al. have identified 11 miRNAs, including miR-21-5p, miR-23-3p, miR-24-3p, miR-25-3p, miR-27a-3p, miR-100-5p, miR-122a-5p, miR-124-3p, miR-125b-5p, miR-148a-3p, and miR-223-3p, were significantly upregulated in the serum and plasma from osteoporotic fracture patients compared with the nonosteoporotic patients' samples through a miRNA array. Except miR-25-3p, miR-27a-3p and miR-223-3p, the other nine miRNAs were subsequently validated their upregulation in the serum of patients with osteoporosis. However, only six miRNAs, miR-21-5p, miR-23-3p, miR-24-3p, miR-25-3p, miR-100-5p, and miR-125b-5p, exhibited a significant upregulation in bone tissue of osteoporotic patients. In total, five miRNAs showed an upregulation both in serum and bone tissue and these five miRNAs have potential acting as biomarkers for osteoporotic diagnosis [79].

Weilner et al. discovered three downregulated miRNAs (miR 133b, miR-328-3p, let-7g-5p) and three upregulated miRNAs (miR-10a-5p, miR-10b-5p, miR-22-3p) in serum from post-menopausal women who recently suffered osteoporotic fractures at the femoral neck relative to control samples. miR-328-3p and let-7g-5p were validated downregulators, subsequently, while miR-22-3p was identified as a significant downregulator in the validation cohort. Moreover, let-7g-5p, miR-10b-5p, miR-100-5p, miR-148a-3p, and miR-21-5p were found to impact osteogenesis through osteogenic differentiation of human mesenchymal stem cells (MSCs) in vitro [80]. Garmilla et al. found miR-187-3p, and miR-518f-3p differentially regulated in osteoporotic bone [81]. Meng et al. found six miRNAs (miR-130b-3p, miR-151a-3p, miR-151b, miR-194-5p, miR-590-5p, and miR-660-5p) were significantly upregulated in the blood of 48 post-menopausal Chinese participants with osteoporosis compared to those with osteopenia. Except miR-660-5p, the other five miRNAs were confirmed the increased expression through external validation in 24 additional post-menopausal women. Moreover, high miRNA expression levels of miR-130b-3p, miR-151a-3p, miR-151b, and miR-194-5p were identified to be significantly correlated with low BMD levels [82].

Taken together, the role of miRNAs as osteoporotic biomarkers mostly attributes to a causal relationship between the miRNA and the osteoblast differentiation [80]. Interestingly, miRNAs, like miR-148a-3p [47] and miR-21-5p [39-42], were found to be involved both in osteogenesis and the regulation of osteoclastogenesis. Further cross validation of cell free blood-based miRNAs with bone miRNAs is strongly relevant. 


\subsection{Potential of miRNAs as Therapeutic Targets in Osteoporosis}

\subsubsection{Transgenic Mice}

Osteoclastic miR-34a-5p-overexpressing transgenic mice showed lower bone resorption and higher bone mass. On the contrary, miR-34a-5p knockout and heterozygous mice exhibited augmented bone resorption and lower bone mass [66].

\subsection{2. miRNA Delivery System}

Through a novel miRNA delivery system based on bacteriophage MS2 virus-like particles (MS2 VLPs), Yao et al. successfully transported miR-146a into human PBMCs, and subsequently demonstrated the inhibitory function of miR-146a-5p in osteoclastogenesis [69]. miR-148a-3p exerts potent inhibitory effects on osteoclast differentiation. Cheng et al. validated the bone mass in mice would increase via a single tail vein injection of a specific antagomiR-148a [47]. Liu et al. established efficient delivery systems to facilitate antagomir-148a-3p to bone resorption surfaces to reduce bone resorption with minimal off-target effects [48]. miR-503-5p acts as a negative regulator of osteoclastogenesis through inhibiting RANK. Ovariectomy (OVX) mice exhibited increased RANK protein expression, promoted bone resorption, and decreased bone mass after using a specific antagomir to silence miR-503-5p expression, whereas agomir-503 exhibit opposite effects [73].

In developing miRNAs-based therapy, it may be helpful to maintain the normal function of osteoblasts if only inhibiting the bone resorption function of osteoclast without affecting the number of osteoclasts. Therefore, it seems that miR-31-5p will be an ideal target, since it just promotes osteoclast function through inhibiting RhoA and RhoA plays a key role in actin ring formation as a small GTPase [44].

\section{Conclusions}

Cumulating evidence suggested that miRNAs are involved in multiple physiological and pathological processes of osteoclast differentiation and function. In general, the expression levels of miRNAs that exert inhibitory effects on osteoclastogenesis tend to decrease during osteoclast formation, and vice versa. MiRNAs-based therapy has been considered as a promising strategy for the treatment of osteoporosis.

Acknowledgments: This work was supported by grants from the National Natural Science Foundation of China (No. 81370969, 81572639); the Ministry of Education of the People's Republic of China (No.20130181110066); the Chengdu Bureau of Science and Technology (No. 2014-HM01-00382-SF).

Author Contributions: Xiao Ji and Xijie Yu designed this review; Xiao Ji and Xiang Chen wrote the manuscript; Xijie Yu revised this manuscript.

Conflicts of Interest: The authors declare no conflict of interest.

\section{Abbreviations}

miRNAs
3'-UTR
OCPs
BRUs
M-CSF
RANKL
NFATc1
MITF
ERK
PI-3K
PU.1

\section{MicroRNAs}

3'-Untranslated Regions

osteoclast precursors

bone remodeling units

macrophage colony-stimulating factor

receptor activator of nuclear factor NF- $\mathrm{B}$ ligand

transcription factor nuclear factor of activated $\mathrm{T}$ cells

microphthalmia-induced transcription factor

extracellular signal-regulated kinase

phosphoinositide 3-kinase

purine-rich binding protein 1 


\begin{tabular}{|c|c|}
\hline RANK & receptor activator of $\mathrm{NF}_{\mathrm{K}} \mathrm{B}$ \\
\hline AP-1 & Activator protein 1 \\
\hline ATF & activating transcription factor \\
\hline MAPK & mitogen-activated protein kinase \\
\hline DC-STAMP & dendritic cell specific transmembrane protein \\
\hline JNK & Janus N-terminal kinase \\
\hline TRAP & tartrate resistant acid phosphatase \\
\hline OSCAR & osteoclast-associated receptor \\
\hline ITAM & Immunoreceptor tyrosine-based activation motif \\
\hline $\mathrm{FcR} \gamma$ & Fc receptor common $\gamma$ subunit \\
\hline DAP12 & DNAX-activating protein 12 \\
\hline TREM-2 & triggering receptor expressed in myeloid cells-2 \\
\hline SIRP $\beta 1$ & signal-regulatory protein $\beta 1$ \\
\hline PIR-A & paired Ig-like receptor-A \\
\hline SNPs & single nucleotide polymorphisms \\
\hline PDCD4 & programmed cell death 4 \\
\hline FasL & Fas ligand \\
\hline BMMs & bone marrow monocytes \\
\hline Calcr & calcitonin receptor \\
\hline Cdc42 & cell division control protein 42 \\
\hline Srgap2 & SLIT-ROBO Rho GTPase-activating protein 2 \\
\hline Gpr85 & G protein-coupled receptor 85 \\
\hline BMD & bone mineral density \\
\hline $\mathrm{HO}-1$ & Heme oxygenase-1 \\
\hline PTEN & Phosphatase and tensin homolog \\
\hline PBMCs & peripheral blood mononuclear cells \\
\hline PIAS & protein inhibitor of activated STAT \\
\hline CTGF/CCN2 & connective tissue growth factor/CCN family 2 \\
\hline Tgif2 & transforming growth factor-b-induced factor 2 \\
\hline RA & rheumatoid arthritis \\
\hline Stat1 & signal transducer and activator transcription 1 \\
\hline OVX & Ovariectomy \\
\hline
\end{tabular}

\section{References}

1. Martin, T.J.; Sims, N.A. Osteoclast-derived activity in the coupling of bone formation to resorption. Trends Mol. Med. 2005, 11, 76-81. [CrossRef] [PubMed]

2. Bartel, D.P. Micrornas: Genomics, biogenesis, mechanism, and function. Cell 2004, 116, 281-297. [CrossRef]

3. Ambros, V. The functions of animal micrornas. Nature 2004, 431, 350-355. [CrossRef] [PubMed]

4. Bartel, D.P. Micrornas: Target recognition and regulatory functions. Cell 2009, 136, 215-233. [CrossRef] [PubMed]

5. Lee, R.C.; Feinbaum, R.L.; Ambros, V. The c. elegans heterochronic gene lin-4 encodes small RNAs with antisense complementarity to lin-14. Cell 1993, 75, 843-854. [CrossRef]

6. Chen, C.Z.; Li, L.; Lodish, H.F.; Bartel, D.P. Micrornas modulate hematopoietic lineage differentiation. Science 2004, 303, 83-86. [CrossRef] [PubMed]

7. Tang, P.; Xiong, Q.; Ge, W.; Zhang, L. The role of microRNAs in osteoclasts and osteoporosis. RNA Biol. 2014, 11, 1355-1363. [CrossRef] [PubMed]

8. Moore, B.T.; Xiao, P. MiRNAs in bone diseases. MicroRNA 2013, 2, 20-31. [CrossRef] [PubMed]

9. Chen, J.; Qiu, M.; Dou, C.; Cao, Z.; Dong, S. MicroRNAs in bone balance and osteoporosis. Drug Dev. Res. 2015, 76, 235-245. [CrossRef] [PubMed]

10. Raisz, L.G. Physiology and pathophysiology of bone remodeling. Clin. Chem. 1999, 45, 1353-1358. [PubMed] 
11. Wilson, S.R.; Peters, C.; Saftig, P.; Bromme, D. Cathepsin K activity-dependent regulation of osteoclast actin ring formation and bone resorption. J. Biol. Chem. 2009, 284, 2584-2592. [CrossRef] [PubMed]

12. Noble, B.S. The osteocyte lineage. Arch. Biochem. Biophys. 2008, 473, 106-111. [CrossRef] [PubMed]

13. Boyce, B.F.; Rosenberg, E.; de Papp, A.E.; Duong, L.T. The osteoclast, bone remodelling and treatment of metabolic bone disease. Eur. J. Clin. Investig. 2012, 42, 1332-1341. [CrossRef] [PubMed]

14. Lagasse, E.; Weissman, I.L. Enforced expression of BCL-2 in monocytes rescues macrophages and partially reverses osteopetrosis in op/op mice. Cell 1997, 89, 1021-1031. [CrossRef]

15. Asagiri, M.; Takayanagi, H. The molecular understanding of osteoclast differentiation. Bone 2007, 40, $251-264$. [CrossRef] [PubMed]

16. Pixley, F.J.; Stanley, E.R. Csf-1 regulation of the wandering macrophage: Complexity in action. Trends Cell Biol. 2004, 14, 628-638. [CrossRef] [PubMed]

17. Ross, F.P.; Teitelbaum, S.L. Av $\beta 3$ and macrophage colony-stimulating factor: Partners in osteoclast biology. Immunol. Rev. 2005, 208, 88-105. [CrossRef] [PubMed]

18. Mellis, D.J.; Itzstein, C.; Helfrich, M.H.; Crockett, J.C. The skeleton: A multi-functional complex organ: The role of key signalling pathways in osteoclast differentiation and in bone resorption. J. Endocrinol. 2011, 211, 131-143. [CrossRef] [PubMed]

19. Scott, E.W.; Simon, M.C.; Anastasi, J.; Singh, H. Requirement of transcription factor PU. 1 in the development of multiple hematopoietic lineages. Science 1994, 265, 1573-1577. [CrossRef] [PubMed]

20. Tondravi, M.M.; McKercher, S.R.; Anderson, K.; Erdmann, J.M.; Quiroz, M.; Maki, R.; Teitelbaum, S.L. Osteopetrosis in mice lacking haematopoietic transcription factor PU.1. Nature 1997, 386, 81-84. [CrossRef] [PubMed]

21. Matsumoto, M.; Kogawa, M.; Wada, S.; Takayanagi, H.; Tsujimoto, M.; Katayama, S.; Hisatake, K.; Nogi, Y. Essential role of p38 mitogen-activated protein kinase in cathepsin $\mathrm{k}$ gene expression during osteoclastogenesis through association of nfatc1 and pu.1. J. Biol. Chem. 2004, 279, 45969-45979. [CrossRef] [PubMed]

22. DeKoter, R.P.; Walsh, J.C.; Singh, H. Pu. 1 regulates both cytokine-dependent proliferation and differentiation of granulocyte/macrophage progenitors. EMBO J. 1998, 17, 4456-4468. [CrossRef] [PubMed]

23. Kwon, O.H.; Lee, C.K.; Lee, Y.I.; Paik, S.G.; Lee, H.J. The hematopoietic transcription factor pu.1 regulates rank gene expression in myeloid progenitors. Biochem. Biophys. Res. Commun. 2005, 335, 437-446. [CrossRef] [PubMed]

24. Weilbaecher, K.N.; Motyckova, G.; Huber, W.E.; Takemoto, C.M.; Hemesath, T.J.; Xu, Y.; Hershey, C.L.; Dowland, N.R.; Wells, A.G.; Fisher, D.E. Linkage of m-csf signaling to mitf, tfe3, and the osteoclast defect in mitfmi/mi mice. Mol. Cell 2001, 8, 749-758. [CrossRef]

25. McGill, G.G.; Horstmann, M.; Widlund, H.R.; Du, J.; Motyckova, G.; Nishimura, E.K.; Lin, Y.L.; Ramaswamy, S.; Avery, W.; Ding, H.F.; et al. Bcl2 regulation by the melanocyte master regulator mitf modulates lineage survival and melanoma cell viability. Cell 2002, 109, 707-718. [CrossRef]

26. Ishii, J.; Kitazawa, R.; Mori, K.; McHugh, K.P.; Morii, E.; Kondo, T.; Kitazawa, S. Lipopolysaccharide suppresses rank gene expression in macrophages by down-regulating pu.1 and mitf. J. Cell. Biochem. 2008, 105, 896-904. [CrossRef] [PubMed]

27. Lu, S.-Y.; Li, M.; Lin, Y.-L. Mitf induction by RANKl is critical for osteoclastogenesis. Mol. Biol. Cell 2010, 21, 1763-1771. [CrossRef] [PubMed]

28. Kim, Y.; Sato, K.; Asagiri, M.; Morita, I.; Soma, K.; Takayanagi, H. Contribution of nuclear factor of activated $\mathrm{t}$ cells $\mathrm{c} 1$ to the transcriptional control of immunoreceptor osteoclast-associated receptor but not triggering receptor expressed by myeloid cells-2 during osteoclastogenesis. J. Biol. Chem. 2005, 280, 32905-32913. [CrossRef] [PubMed]

29. Obata, T.; Brown, G.E.; Yaffe, M.B. Map kinase pathways activated by stress: The p38 MAPK pathway. Crit. Care Med. 2000, 28, N67-N77. [CrossRef] [PubMed]

30. Wagner, E.F.; Eferl, R. Fos/ap-1 proteins in bone and the immune system. Immunol. Rev. 2005, 208, $126-140$. [CrossRef] [PubMed]

31. Takayanagi, H.; Kim, S.; Koga, T.; Nishina, H.; Isshiki, M.; Yoshida, H.; Saiura, A.; Isobe, M.; Yokochi, T.; Inoue, J.; et al. Induction and activation of the transcription factor NFATc1 (NFAT2) integrate RANK1 signaling in terminal differentiation of osteoclasts. Dev. Cell 2002, 3, 889-901. [CrossRef] 
32. Kim, K.; Kim, J.H.; Lee, J.; Jin, H.M.; Lee, S.H.; Fisher, D.E.; Kook, H.; Kim, K.K.; Choi, Y.; Kim, N. Nuclear factor of activated $\mathrm{t}$ cells $\mathrm{c} 1$ induces osteoclast-associated receptor gene expression during tumor necrosis factor-related activation-induced cytokine-mediated osteoclastogenesis. J. Biol. Chem. 2005, 280, 35209-35216. [CrossRef] [PubMed]

33. Crotti, T.N.; Flannery, M.; Walsh, N.C.; Fleming, J.D.; Goldring, S.R.; McHugh, K.P. NFATc1 regulation of the human $\beta 3$ integrin promoter in osteoclast differentiation. Gene 2006, 372, 92-102. [CrossRef] [PubMed]

34. Koga, T.; Inui, M.; Inoue, K.; Kim, S.; Suematsu, A.; Kobayashi, E.; Iwata, T.; Ohnishi, H.; Matozaki, T.; Kodama, T.; et al. Costimulatory signals mediated by the itam motif cooperate with rankl for bone homeostasis. Nature 2004, 428, 758-763. [CrossRef] [PubMed]

35. Marcelis, C.L.; Hol, F.A.; Graham, G.E.; Rieu, P.N.; Kellermayer, R.; Meijer, R.P.; Lugtenberg, D.; Scheffer, H.; van Bokhoven, H.; Brunner, H.G.; et al. Genotype-phenotype correlations in MYCN-related feingold syndrome. Hum. Mutat. 2008, 29, 1125-1132. [CrossRef] [PubMed]

36. Han, Y.C.; Vidigal, J.A.; Mu, P.; Yao, E.; Singh, I.; Gonzalez, A.J.; Concepcion, C.P.; Bonetti, C.; Ogrodowski, P.; Carver, B.; et al. An allelic series of miR-17 approximately 92-mutant mice uncovers functional specialization and cooperation among members of a microrna polycistron. Nat. Genet. 2015, 47, 766-775. [CrossRef] [PubMed]

37. Dole, N.S.; Delany, A.M. Microrna variants as genetic determinants of bone mass. Bone 2016, 84, 57-68. [CrossRef] [PubMed]

38. Franceschetti, T.; Dole, N.S.; Kessler, C.B.; Lee, S.K.; Delany, A.M. Pathway analysis of microRNA expression profile during murine osteoclastogenesis. PLoS ONE 2014, 9, e107262. [CrossRef] [PubMed]

39. Sugatani, T.; Vacher, J.; Hruska, K.A. A microRNA expression signature of osteoclastogenesis. Blood 2011, 117, 3648-3657. [CrossRef] [PubMed]

40. Fujita, S.; Ito, T.; Mizutani, T.; Minoguchi, S.; Yamamichi, N.; Sakurai, K.; Iba, H. miR-21 gene expression triggered by ap-1 is sustained through a double-negative feedback mechanism. J. Mol. Biol. 2008, 378, 492-504. [CrossRef] [PubMed]

41. Garcia Palacios, V.; Robinson, L.J.; Borysenko, C.W.; Lehmann, T.; Kalla, S.E.; Blair, H.C. Negative regulation of RANKl-induced osteoclastic differentiation in raw264.7 cells by estrogen and phytoestrogens. J. Biol. Chem. 2005, 280, 13720-13727. [CrossRef] [PubMed]

42. Sugatani, T.; Hruska, K.A. Down-regulation of miR-21 biogenesis by estrogen action contributes to osteoclastic apoptosis. J. Cell. Biochem. 2013, 114, 1217-1222. [CrossRef] [PubMed]

43. Franceschetti, T.; Kessler, C.B.; Lee, S.K.; Delany, A.M. miR-29 promotes murine osteoclastogenesis by regulating osteoclast commitment and migration. J. Cell. Biochem. 2013, 288, 33347-33360. [CrossRef] [PubMed]

44. Mizoguchi, F.; Murakami, Y.; Saito, T.; Miyasaka, N.; Kohsaka, H. miR-31 controls osteoclast formation and bone resorption by targeting RHOA. Arthritis Res. Ther. 2013, 15, R102. [CrossRef] [PubMed]

45. Wang, Y.; Li, L.; Moore, B.T.; Peng, X.H.; Fang, X.; Lappe, J.M.; Recker, R.R.; Xiao, P. miR-133a in human circulating monocytes: A potential biomarker associated with postmenopausal osteoporosis. PLoS ONE 2012, 7, e34641. [CrossRef] [PubMed]

46. Cao, Z.; Moore, B.T.; Wang, Y.; Peng, X.H.; Lappe, J.M.; Recker, R.R.; Xiao, P. miR-422a as a potential cellular microRNA biomarker for postmenopausal osteoporosis. PLoS ONE 2014, 9, e97098. [CrossRef] [PubMed]

47. Cheng, P.; Chen, C.; He, H.B.; Hu, R.; Zhou, H.D.; Xie, H.; Zhu, W.; Dai, R.C.; Wu, X.P.; Liao, E.Y.; et al. miR-148a regulates osteoclastogenesis by targeting v-maf musculoaponeurotic fibrosarcoma oncogene homolog b. J. Bone Miner. Res. 2013, 28, 1180-1190. [CrossRef] [PubMed]

48. Liu, J.; Dang, L.; Li, D.; Liang, C.; He, X.; Wu, H.; Qian, A.; Yang, Z.; Au, D.W.; Chiang, M.W.; et al. A delivery system specifically approaching bone resorption surfaces to facilitate therapeutic modulation of microRNAs in osteoclasts. Biomaterials 2015, 52, 148-160. [CrossRef] [PubMed]

49. Ke, K.; Sul, O.J.; Rajasekaran, M.; Choi, H.S. MicroRNA-183 increases osteoclastogenesis by repressing heme oxygenase-1. Bone 2015, 81, 237-246. [CrossRef] [PubMed]

50. Wang, X.; Guo, B.; Li, Q.; Peng, J.; Yang, Z.; Wang, A.; Li, D.; Hou, Z.; Lv, K.; Kan, G.; et al. miR-214 targets atf4 to inhibit bone formation. Nat. Med. 2013, 19, 93-100. [CrossRef] [PubMed]

51. Zhao, C.; Sun, W.; Zhang, P.; Ling, S.; Li, Y.; Zhao, D.; Peng, J.; Wang, A.; Li, Q.; Song, J.; et al. miR-214 promotes osteoclastogenesis by targeting pten/pi3k/akt pathway. RNA Biol. 2015, 12, 343-353. [CrossRef] [PubMed] 
52. Sugatani, T.; Alvarez, U.; Hruska, K.A. Pten regulates rankl- and osteopontin-stimulated signal transduction during osteoclast differentiation and cell motility. J. Biol. Chem. 2003, 278, 5001-5008. [CrossRef] [PubMed]

53. Jang, H.D.; Noh, J.Y.; Shin, J.H.; Lin, J.J.; Lee, S.Y. Pten regulation by the akt/gsk-3 $\beta$ axis during rank1 signaling. Bone 2013, 55, 126-131. [CrossRef] [PubMed]

54. Kagiya, T.; Nakamura, S. Expression profiling of microRNAs in raw264.7 cells treated with a combination of tumor necrosis factor alpha and rankl during osteoclast differentiation. J. Periodontal Res. 2013, 48, 373-385. [CrossRef] [PubMed]

55. Ell, B.; Kang, Y. MicroRNAs as regulators of bone homeostasis and bone metastasis. Bone Key Rep. $2014,3$. [CrossRef] [PubMed]

56. Sugatani, T.; Hruska, K.A. Impaired micro-RNA pathways diminish osteoclast differentiation and function. J. Biol. Chem. 2009, 284, 4667-4678. [CrossRef] [PubMed]

57. Sugatani, T.; Hruska, K.A. MicroRNA-223 is a key factor in osteoclast differentiation. J. Cell. Biochem. 2007, 101, 996-999. [CrossRef] [PubMed]

58. Bunt, J.; Lim, J.W.; Zhao, L.; Mason, S.; Richards, L.J. Pax6 does not regulate NFIA and NFIB expression during neocortical development. Sci. Rep. 2015, 5, 10668. [CrossRef] [PubMed]

59. O'Connell, R.M. MicroRNAs function on a new level. Blood 2012, 119, 3875-3876. [CrossRef] [PubMed]

60. Fazi, F.; Rosa, A.; Fatica, A.; Gelmetti, V.; de Marchis, M.L.; Nervi, C.; Bozzoni, I. A minicircuitry comprised of microRNA-223 and transcription factors NFI-A and c/EBP $\alpha$ regulates human granulopoiesis. Cell 2005, 123, 819-831. [CrossRef] [PubMed]

61. Shibuya, H.; Nakasa, T.; Adachi, N.; Nagata, Y.; Ishikawa, M.; Deie, M.; Suzuki, O.; Ochi, M. Overexpression of microRNA-223 in rheumatoid arthritis synovium controls osteoclast differentiation. Modern Rheumatol. 2013, 23, 674-685. [CrossRef]

62. Fukao, T.; Fukuda, Y.; Kiga, K.; Sharif, J.; Hino, K.; Enomoto, Y.; Kawamura, A.; Nakamura, K.; Takeuchi, T.; Tanabe, M. An evolutionarily conserved mechanism for microRNA-223 expression revealed by microRNA gene profiling. Cell 2007, 129, 617-631. [CrossRef] [PubMed]

63. Liu, T.; Qin, A.P.; Liao, B.; Shao, H.G.; Guo, L.J.; Xie, G.Q.; Yang, L.; Jiang, T.J. A novel microrna regulates osteoclast differentiation via targeting protein inhibitor of activated stat3 (PIAS3). Bone 2014, 67, 156-165. [CrossRef] [PubMed]

64. Dou, C.; Zhang, C.; Kang, F.; Yang, X.; Jiang, H.; Bai, Y.; Xiang, J.; Xu, J.; Dong, S. miR-7b directly targets dc-stamp causing suppression of nfatc1 and c-fos signaling during osteoclast fusion and differentiation. Biochim. Biophys. Acta 2014, 1839, 1084-1096. [CrossRef] [PubMed]

65. Kim, K.; Kim, J.H.; Kim, I.; Lee, J.; Seong, S.; Park, Y.W.; Kim, N. MicroRNA-26a regulates rankl-induced osteoclast formation. Mol. Cells 2015, 38, 75-80. [PubMed]

66. Krzeszinski, J.Y.; Wei, W.; Huynh, H.; Jin, Z.; Wang, X.; Chang, T.C.; Xie, X.J.; He, L.; Mangala, L.S.; Lopez-Berestein, G.; et al. miR-34a blocks osteoporosis and bone metastasis by inhibiting osteoclastogenesis and tgif2. Nature 2014, 512, 431-435. [CrossRef] [PubMed]

67. Lee, Y.; Kim, H.J.; Park, C.K.; Kim, Y.G.; Lee, H.J.; Kim, J.Y.; Kim, H.H. MicroRNA-124 regulates osteoclast differentiation. Bone 2013, 56, 383-389. [CrossRef] [PubMed]

68. Guo, L.J.; Liao, L.; Yang, L.; Li, Y.; Jiang, T.J. miR-125a TNF receptor-associated factor 6 to inhibit osteoclastogenesis. Exp. Cell Res. 2014, 321, 142-152. [CrossRef] [PubMed]

69. Nakasa, T.; Shibuya, H.; Nagata, Y.; Niimoto, T.; Ochi, M. The inhibitory effect of microRNA-146a expression on bone destruction in collagen-induced arthritis. Arthritis Rheum. 2011, 63, 1582-1590. [CrossRef] [PubMed]

70. Lu, L.-F.; Boldin, M.P.; Chaudhry, A.; Lin, L.-L.; Taganov, K.D.; Hanada, T.; Yoshimura, A.; Baltimore, D.; Rudensky, A.Y. Function of miR-146a in controlling treg cell-mediated regulation of th1 responses. Cell 2010, 142, 914-929. [CrossRef] [PubMed]

71. Yao, Y.; Jia, T.; Pan, Y.; Gou, H.; Li, Y.; Sun, Y.; Zhang, R.; Zhang, K.; Lin, G.; Xie, J. Using a novel microRNA delivery system to inhibit osteoclastogenesis. Int. J. Mol. Sci. 2015, 16, 8337-8350. [CrossRef] [PubMed]

72. Qu, B.; Xia, X.; Yan, M.; Gong, K.; Deng, S.; Huang, G.; Ma, Z.; Pan, X. miR-218 is involved in the negative regulation of osteoclastogenesis and bone resorption by partial suppression of p38MAPK-c-Fos-NFATc1 signaling: Potential role for osteopenic diseases. Exp. Cell Res. 2015, 338, 89-96. [CrossRef] [PubMed]

73. Chen, C.; Cheng, P.; Xie, H.; Zhou, H.D.; Wu, X.P.; Liao, E.Y.; Luo, X.H. miR-503 regulates osteoclastogenesis via targeting rank. J. Bone Miner. Res. 2014, 29, 338-347. [CrossRef] [PubMed] 
74. Woo, S.-B.; Hellstein, J.W.; Kalmar, J.R. Systematic review: Bisphosphonates and osteonecrosis of the jaws. Ann. Intern. Med. 2006, 144, 753-761. [CrossRef] [PubMed]

75. Black, D.M.; Delmas, P.D.; Eastell, R.; Reid, I.R.; Boonen, S.; Cauley, J.A.; Cosman, F.; Lakatos, P.; Leung, P.C.; Man, Z.; et al. Once-yearly zoledronic acid for treatment of postmenopausal osteoporosis. N. Engl. J. Med. 2007, 356, 1809-1822. [CrossRef] [PubMed]

76. Odvina, C.V.; Zerwekh, J.E.; Rao, D.S.; Maalouf, N.; Gottschalk, F.A.; Pak, C.Y. Severely suppressed bone turnover: A potential complication of alendronate therapy. J. Clin. Endocrinol. Metab. 2005, 90, 1294-1301. [CrossRef] [PubMed]

77. Panach, L.; Mifsut, D.; Tarín, J.J.; Cano, A.; García-Pérez, M.Á. Serum circulating microRNAs as biomarkers of osteoporotic fracture. Calcif. Tissue Int. 2015, 97, 495-505. [CrossRef] [PubMed]

78. Li, H.; Wang, Z.; Fu, Q.; Zhang, J. Plasma miRNA levels correlate with sensitivity to bone mineral density in postmenopausal osteoporosis patients. Biomarkers 2014, 19, 553-556. [CrossRef] [PubMed]

79. Seeliger, C.; Karpinski, K.; Haug, A.T.; Vester, H.; Schmitt, A.; Bauer, J.S.; van Griensven, M. Five freely circulating mirnas and bone tissue miRNAs are associated with osteoporotic fractures. J. Bone Miner. Res. 2014, 29, 1718-1728. [CrossRef] [PubMed]

80. Weilner, S.; Skalicky, S.; Salzer, B.; Keider, V.; Wagner, M.; Hildner, F.; Gabriel, C.; Dovjak, P.; Pietschmann, P.; Grillari-Voglauer, R. Differentially circulating miRNAs after recent osteoporotic fractures can influence osteogenic differentiation. Bone 2015, 79, 43-51. [CrossRef] [PubMed]

81. Garmilla-Ezquerra, P.; Sañudo, C.; Delgado-Calle, J.; Pérez-Nuñez, M.I.; Sumillera, M.; Riancho, J.A. Analysis of the bone micrornome in osteoporotic fractures. Calcif. Tissue Int. 2015, 96, 30-37. [CrossRef] [PubMed]

82. Meng, J.; Zhang, D.; Pan, N.; Sun, N.; Wang, Q.; Fan, J.; Zhou, P.; Zhu, W.; Jiang, L. Identification of miR-194-5p as a potential biomarker for postmenopausal osteoporosis. Peer J 2015, 3, e971. [CrossRef] [PubMed]

(C) 2016 by the authors; licensee MDPI, Basel, Switzerland. This article is an open access article distributed under the terms and conditions of the Creative Commons by Attribution (CC-BY) license (http://creativecommons.org/licenses/by/4.0/). 